\title{
LAMB2-related infantile-onset nephrotic syndrome
}

INSERM

\section{Source}

INSERM. (1999). Orphanet: an online rare disease and orphan drug data base. LAMB2related infantile-onset nephrotic syndrome. ORPHA:306507

LAMB2-related infantile-onset nephrotic syndrome is a rare primary glomerular disease due to homozygous mutations in LAMB2 gene, characterized by prenatal or early-onset progressive steroid-resistant nephrotic syndrome leading to renal failure, and variable ocular defects including myopia, fundus abnormalities, strabismus or nystagmus, without severe visual impairment or blindness. Patients present in early infancy with massive proteinuria, edema, hypertension, and hyperlipidemia. Psychomotor development is normal. 